\title{
S100A16 suppresses the proliferation, migration and invasion of colorectal cancer cells in part via the JNK/p38 MAPK pathway
}

\author{
SHIYU OU ${ }^{1,2}$, YAN LIAO $^{1}$, JIE SHI $^{1}$, JING TANG $^{3}$, YANQING YE ${ }^{1}$, FENGFEI WU $^{1}$, \\ WEIDONG WANG ${ }^{1}$, JIEYING FEI ${ }^{1}$, FANG XIE $^{1}$ and LAN BAI ${ }^{1}$
}

\begin{abstract}
${ }^{1}$ Guangdong Provincial Key Laboratory of Gastroenterology, Department of Gastroenterology, Nanfang Hospital, Southern Medical University, Guangzhou, Guangdong 510515; ${ }^{2}$ Department of Gastroenterology, The Fourth Affiliated Hospital of Guangxi Medical University, Liuzhou, Guangxi 545005; ${ }^{3}$ Department of Gastroenterology, The First Affiliated Hospital of Guangxi Medical University, Nanning, Guangxi 530021, P.R. China
\end{abstract}

Received March 26, 2020; Accepted November 26, 2020

DOI: $10.3892 / \mathrm{mmr} .2020 .11803$

\begin{abstract}
S100 calcium binding protein A16 (S100A16) is the most recent member of the $\mathrm{S} 100$ calcium-binding protein family. The function of S100A16 has been associated with various types of cancer; however, its role in colorectal cancer (CRC) remains unknown. Therefore, the aim of the present study was to investigate the role of S100A16 in CRC progression. The Oncomine dataset used in the current study revealed that the expression of S100A16 was decreased in CRC compared with normal colorectal tissues. Similar results were also determined via immunohistochemistry. In addition, a negative association was identified between S100A16 expression and the prognosis of patients with CRC. Further functional experiments revealed that S100A16 knockdown promoted the proliferation, migration and invasion of HCT116 and SW480 cells, and vice versa in Lovo cells. Epithelial-mesenchymal transition (EMT) was promoted and the JNK/p38 MAPK pathway was activated in HCT116 cells following S100A16 knockdown, as determined via western blotting. Furthermore, S100A16 silencing promoted the migration and invasion of cells. EMT was also reversed when cells were treated with the JNK inhibitor (SP600125) or the p38 inhibitor (SB203580). In summary, the results of the present study demonstrated that S100A16 suppressed the proliferation, migration and invasion of CRC cells partially via the JNK/p38 MAPK signalling pathway and subsequent EMT mediation.
\end{abstract}

Correspondence to: Professor Lan Bai, Guangdong Provincial Key Laboratory of Gastroenterology, Department of Gastroenterology, Nanfang Hospital, Southern Medical University, 1838 North Guangzhou Avenue, Guangzhou, Guangdong 510515, P.R. China E-mail: oushiyu2012@smu.edu.cn

Key words: S100 calcium binding protein A16, colorectal cancer, tumour metastasis, epithelial-mesenchymal transition

\section{Introduction}

Colorectal cancer (CRC) is one of the most common cancer types and is the second cause of cancer-associated mortality worldwide, causing about 600,000 deaths per year (1). Despite progress in diagnostic and therapeutic techniques, the overall survival rate of patients with CRC remains poor, due to diagnosis occurring at a later stage, where metastasis has developed $(2,3)$. Therefore, it is important to gain a greater understanding of the underlying molecular mechanisms involved in CRC, which will contribute to the early diagnosis and effective treatment of the disease.

S100 calcium binding protein A16 (S100A16) is a member of the S100 protein family, which is comprised of acidic proteins with an EF-hand $\mathrm{Ca}^{2+}$ binding motif (4). S100 proteins serve a variety of regulatory functions in cellular processes, such as motility, differentiation, cell proliferation, contraction, transcription, cell cycle processes and secretion (5). The abnormal protein expression of S100 has been exhibited in several types of tumour and is considered to be a potential marker of cancer $(6,7)$. As the most recent member of the S100 family, S100A16 has been implicated in numerous human pathophysiological processes, such as inflammation, adipogenesis, osteoporosis and tumour progression (8-10). In addition, previous studies have reported that the function of S100A16 in cancer is complex (11-14). For instance, S100A16 demonstrates a variable effect in tumour cells of different tissues. Several studies have reported that S100A16 is associated with tumour progression in bladder, lung and breast cancer (11-13). However, an additional study reported the tumour-suppressive function of S100A16 in oral squamous cell carcinoma (14). Moreover, the abnormal expression of S100A16 is involved in CRC progression. It has also been shown that the low membrane expression of S100A16 is associated with a poor prognosis in patients with CRC, potentially serving as a suppressor of cancer (15). However, the specific modulatory role of S100A16 in CRC is yet to be elucidated.

The aim of the present study was to investigate whether S100A16 could inhibit the progression of CRC by regulating JNK/p38 MAPK signalling pathway, which may provide 
insight into the role of S100A16 in CRC as a potential prognostic marker of patients with CRC.

\section{Materials and methods}

Data collection and analysis. The data of 45 colorectal adenocarcinoma and 24 normal colorectal tissues were downloaded from the GSE20916 microarray dataset (http://www.ncbi.nlm. nih.gov/geo/query/acc.cgi?acc=GSE20916) (16). The mRNA expression values of S100A16 in colorectal adenocarcinoma and normal colorectal tissues were analyzed on the Oncomine platform (https://www.oncomine.org/), which collects publicly available cancer microarray data. The term 'S100A16' was searched on Oncomine. 'Cancer type' and 'analysis type' were then defined as 'colorectal cancer' and 'cancer vs. normal analysis', respectively. The cut-off of P-value and fold change were 0.05 and 2 , respectively. The data were also analysed with an unpaired-t test between two groups using GraphPad Prism 6 (GraphPad Software, Inc.).

Tissue samples and microarrays. A total of 10 CRC samples and paired colorectal normal tissues were obtained from patients (age range, 31-71 years; 6 male and 4 female patients) admitted to the Department of Gastrointestinal Surgery, Nanfang Hospital, Southern Medical University between January 2015 and June 2019. All patients provided written informed consent and the study protocol was approved by the Nanfang Hospital Institutional Review Board (approval no. NFEC-2017-147). In addition, microarrays of CRC tissue comprising 100 cases (60 cases of CRC samples with matched, normal colorectal tissue samples; 40 cases were CRC samples alone.) were purchased from Shanghai Outdo Biotech Co., Ltd. All tumours were diagnosed as colorectal adenocarcinoma and all specimens were used for routine pathological processing. Out of all 110 specimens used in the present study, 104 had complete clinicopathological features and complete follow-up data.

Cell culture and treatment. Human CRC cell lines, including SW480, SW620, HCT116 and Lovo, were obtained from The Cell Bank of Type Culture Collection of Chinese Academy of Sciences and identified by the American Type Culture Collection. RKO cells were obtained from the American Type Culture Collection. All cells were cultured in medium containing 90\% DMEM (HyClone; Cytiva) and 10\% FBS (HyClone; Cytiva) in a $5 \% \mathrm{CO}_{2}$ atmosphere at $37^{\circ} \mathrm{C}$.

To determine the impact of the JNK or p38 MAPK pathway, $10 \mu \mathrm{M}$ JNK inhibitor SP600125 (cat. no. 8177; Cell Signaling Technology, Inc.) or $10 \mu \mathrm{M}$ p38 inhibitor SB203580 (cat. no. 5633; Cell Signaling Technology, Inc.) were applied to $\mathrm{HCT} 116$ cells and incubated at $37^{\circ} \mathrm{C}$ with $5 \% \mathrm{CO}_{2}$ for $48 \mathrm{~h}$.

Immunohistochemistry (IHC) staining. Tissue samples were fixed with $4 \%$ paraformaldehyde for 2 days at room temperature and embedded in paraffin, after which $4-\mu \mathrm{m}$ were cut and mounted onto slides. Slides were incubated at $56^{\circ} \mathrm{C}$, deparaffinised in xylene and dehydrated in a graded series of alcohol. Heat-induced antigen retrieval was carried out with sodium citrate $(\mathrm{pH} 6.0)$ in a microwaving at $100^{\circ} \mathrm{C}$ for $30 \mathrm{~min}$, IHC staining was performed as previously described (17).
Briefly, endogenous peroxidase activity was inhibited using $3 \%$ hydrogen peroxide for $10 \mathrm{~min}$ at room temperature. After blocking with 5\% goat serum (cat. no. BMS0050; Abbkine Scientific Co., Ltd.) diluted with PBS at room temperature for $30 \mathrm{~min}$, sections were incubated with anti-S100A16 antibodies (cat. no. 11456-1-AP; 1:300; ProteinTech Group, Inc.) at $4^{\circ} \mathrm{C}$ overnight. Samples were then incubated with HRP-conjugated secondary antibodies (cat. no. ab5879; Abcam) at room temperature for $30 \mathrm{~min}$. The resulting protein was presented as a brown pigment using the standard diaminodianiline method (cat. no. SAP 9101; OriGene Technologies, Inc.). Slides were subsequently double-stained with haematoxylin at room temperature for $20 \mathrm{sec}$, after which immunostaining was assessed using an Olympus BX-53 microscope (Olympus Corporation; magnification, x100-400). The degree of IHC staining was evaluated as previously described $(0$, no staining; 1 , weak staining; 2 , moderate staining; and 3, strong staining) (18). Scores of 0-1 were defined as low expression, and scores of 2-3 were defined as high expression. The immunoreactivity score was determined as follows: Immunoreactivity score $=$ the percentage of positive staining $\mathrm{x}$ the staining intensity score.

Overexpression and knockdown of S100A16. Based on the S100A16 gene sequence in GenBank (NM_080388; https://www.ncbi.nlm.nih.gov/nuccore), primers specifically matching S100A16 mRNA were constructed, which were amplified, cut by HindIII and BamHI and then sub-cloned into pcDNA3.1 vector (cat. no. P0157; www.miaolingbio.com) or GV287-EGFP lentiviral vector (Shanghai GeneChem Co., Ltd.) to overexpress S100A16 after screening. The empty lentiviral vector was used as a negative control.

To overexpress S100A16, colon cancer cells were transfected with $5 \mathrm{nM} \mathrm{S100A16}$ pcDNA3.1(+) vector using Lipofectamine ${ }^{\circledR} 2000$ reagent (Thermo Fisher Scientific, Inc.) according to the manufacturer's instructions for two days at $37^{\circ} \mathrm{C}$. After $48 \mathrm{~h}$ of transfection, the expression of S100A16 was determined via western blotting, and the transfected cells were collected for subsequent experiments in vitro.

For lentivirus transduction, 7.5 $\mu \mathrm{g} \mathrm{GV287-EGFP-S100A16}$ (lenti-S100A16) lentiviral vector (Shanghai GeneChem Co., Ltd.), mixed with $5 \mu \mathrm{g}$ pMD2.G (cat. no. p2062; www. miaolingbio.com) and $10 \mu \mathrm{g}$ psPAX2 (cat. no. p2061; www. miaolingbio.com), were co-transfected into 293T cells (The Cell Bank of Type Culture Collection of Chinese Academy of Sciences) with Lipofectamine ${ }^{\circledR} 2000$ reagent (Thermo Fisher Scientific, Inc.) according to the manufacturer's instructions at $37^{\circ} \mathrm{C}$ with $5 \% \mathrm{CO}_{2}$ for $48 \mathrm{~h}$. The viral supernatants were collected, centrifuged at $4000 \mathrm{x}$ g for $10 \mathrm{~min}$ at $4^{\circ} \mathrm{C}$, then filtered through $0.45-\mu \mathrm{m}$ PVDF membranes. The virus concentration was determined by reverse transcriptionquantitative PCR to measure the number of integrated copies of 293 T cells. Lentivirus (lentivirus-S100A16) were then used to infect colon cancer cells using $5 \mu \mathrm{g} / \mathrm{ml}$ polybrene (Hanbio Biotechnology Co., Ltd.) with an MOI of 5. The infected cells were cultured with puromycin $(5 \mu \mathrm{g} / \mathrm{ml})$ for 14 days at $37^{\circ} \mathrm{C}$ to establish stably infected cells for mouse xenografts.

To knockdown S100A16, CRC cells were transfected with $50 \mathrm{nM}$ S100A16 small interfering RNA (siRNA/siS100A16; Shanghai GenePharma Co., Ltd.) using Lipofectamine 
2000 reagent (Thermo Fisher Scientific, Inc.) according to the manufacturer's instructions for 2 days at $37^{\circ} \mathrm{C}$. The following sequences were utilized: si-S100A16\#1, sense, 5'-GCAUCA GCUUCGAUGAGUATT-3' and antisense, 5'-UACUCAUCGAAGCUGAUGCTT-3'; si-S100A16\#2, sense, 5'-CCAGAACCUGGAUGCCAAUTT-3' and antisense, 5'-AUUGGCAUCCAGGUUCUGGTT-3'; si-S100A16\#3, sense, 5'-GCUGGAGAAGGCAGUCAUUTT-3' and antisense, 5'-AAUGACUGCCUUCUCCAGCTT-3'; negative control, sense, 5'-UUCUCCGAACGUGUCACGUTT-3' and antisense, 5'-ACGUGACACGUUCGGAGAATT-3'. At $48 \mathrm{~h}$ following transfection, the expression of S100A16 was determined via western blotting, and the transfected cells were collected for subsequent experiments.

Cell proliferation assay. After S100A16 siRNA was transfected into SW480 and HCT116 cells or following S100A16 pcDNA3.1(+) vector transfection into Lovo cells, CRC cells $\left(1 \times 10^{3}\right.$ cells/well) were seeded into 96 -well plates. Cells were cultured for $24,48,72,96$ and $120 \mathrm{~h}$, after which $10 \mu \mathrm{l}$ Cell Counting Kit-8 (CCK-8) solution (Dojindo Molecular Technologies, Inc.) was added and incubated in a $5 \% \mathrm{CO}_{2}$ atmosphere at $37^{\circ} \mathrm{C}$ for an additional $4 \mathrm{~h}$. The kit was used according to the manufacturer's instructions. Optical density was measured at $450 \mathrm{~nm}$ using an automatic enzyme standard.

Cell migration and invasion assays. Cell migration and invasion assays were performed using a Transwell system (Corning, Inc.). For cell invasion, an $8 \mu \mathrm{m}$-pore polycarbonate membrane was coated with Matrigel (BD Biosciences) at $37^{\circ} \mathrm{C}$ for $5 \mathrm{~h}$. Cells at density of $2.5 \times 10^{4}$ (migration) and $5 \times 10^{4}$ (invasion) per well were suspended in $500 \mu \mathrm{l}$ serum-free medium and subsequently added to the upper chamber. A total of $600 \mu \mathrm{l}$ medium containing $10 \%$ FBS was added to the lower chamber. After 24 (migration) or $48 \mathrm{~h}$ (invasion) incubation at $37^{\circ} \mathrm{C}$, migrated or invaded cells in the lower chambers were stained with $0.1 \%$ crystal violet at room temperature for $30 \mathrm{~min}$ and counted using light microscopy (magnification, x100).

$R N A$ isolation and reverse transcription-quantitative PCR $(R T-q P C R)$. Total RNA was extracted from the harvested cells using TRIzol ${ }^{\circledR}$ (Thermo Fisher Scientific, Inc.) according to the manufacturer's protocol. First-Strand cDNA Synthesis was carried out by reverse-transcription using PrimeScript ${ }^{\mathrm{TM}}$ RT Master Mix (Takara) and qPCR was performed with a LightCycler $480^{\circledR}$ System (Roche Diagnostics) using SYBRGreen mix (Takara Bio, Inc.), according to the manufacturer's instructions. The thermocycling conditions were as follows: i) $94^{\circ} \mathrm{C}$ for $5 \mathrm{~min}$; ii) 40 amplification cycles at $94^{\circ} \mathrm{C}$ for $30 \mathrm{sec}$, $57^{\circ} \mathrm{C}$ for $30 \mathrm{sec}$ and $72^{\circ} \mathrm{C}$ for $30 \mathrm{sec}$; and iii) $72^{\circ} \mathrm{C}$ for $5 \mathrm{~min}$. The primer sequences used for PCR were as follows: i) S100A16 forward, 5'-TTTTGTCCATCTCCTTTCACCA-3' and reverse, 5'-CAGGCAAATCAGACTCCCTTC-3'; ii) S100A4 forward, 5-GTACTCGGGCAAAGAGGGTG-3' and reverse, 5'-TTGTCCCTGTTGCTGTCCAA-3'; iii) S100A14 forward, 5'-TGCTCTAGAATGGGACAGTGTCGGTCAGCC-3' and reverse, 5'-CGCGGATCCTCAGTGCCCCCGGA CAGGCCT-3'; and iv) GAPDH (the internal reference) forward, 5'-CTGGGCTACACTGAGCACC-3' and reverse,
5'-AAGTGGTCGTTGAGGGCAATG-3'. Relative gene expression was determined using the $2^{-\Delta \Delta \mathrm{Cq}}$ method (19).

Western blotting. Cultured cells were lysed in RIPA buffer containing $1 \mathrm{X}$ protease cocktail inhibitor (Sigma-Aldrich; Merck KGaA). Protein concentrations were determined using a BCA protein assay kit (Thermo Fisher Scientific, Inc.). A total of $30 \mu \mathrm{g}$ proteins from different groups were then separated on 10-12\% SDS-polyacrylamide gels for electrophoresis. Separated proteins were transferred to PVDF membranes (EMD Millipore). After being blocked with non-fat milk for $1 \mathrm{~h}$ at room temperature, the membranes were incubated with the following primary antibodies at $4^{\circ} \mathrm{C}$ overnight: anti-rabbit S100A4 (cat. no. 16105-1-AP; 1:500; ProteinTech Group, Inc.), anti-rabbit S100A14 (cat. no. 10489-1-AP; 1:500; ProteinTech Group, Inc.), anti-rabbit S100A16 (cat. no. 11456-1-AP; 1:500; ProteinTech Group, Inc.) anti-phosphorylated (p)-rabbit JNK (cat. no. 9255; Cell Signaling Technology, Inc.), anti-rabbit JNK (cat. no. 9252; Cell Signaling Technology, Inc.), anti-prabbit ERK1/2 (cat. no. 4370; Cell Signaling Technology, Inc.), anti-rabbit ERK1/2 (cat. no. 4695; Cell Signaling Technology, Inc.), anti-p-rabbit p38 MAPK (cat. no. 9216; Cell Signaling Technology, Inc.), anti-rabbit p38 MAPK (cat. no. 9212; Cell Signaling Technology, Inc.), anti-rabbit vimentin (cat. no. 12826; Cell Signaling Technology, Inc.), anti-mouse E-cadherin (cat. no. 14472; Cell Signaling Technology, Inc.), anti-mouse N-cadherin (cat. no. 14215; Cell Signaling Technology, Inc.) and anti-mouse GAPDH (cat. no. 51332; Cell Signaling Technology, Inc.). After incubation with horseradish peroxidase-conjugated secondary antibodies (cat. nos. 7074 and 7076; Cell Signaling Technology, Inc.) for $1 \mathrm{~h}$ at room temperature, immunoreactive proteins were visualized using chemiluminescence detection reagents (EMD Millipore) using the FluorChem E system (ProteinSimple). Blots were semi-quantified using AlphaView SA software (ProteinSimple; version 3.4.0.0). The grayscale values of protein bands were normalized to that of GAPDH.

Mouse xenografts of CRC cells. A total of $10 \mathrm{BALB} / \mathrm{c}$ nude male mice (weight, 12-14 g; age, 4-6 weeks) were purchased from Hunan SJA Laboratory Animal Co., Ltd. The mice were fed with common feed and sterile water ad libitum and housed in $21-23^{\circ} \mathrm{C}$ with $54-56 \%$ humidity and a 12 -h light/dark cycle. Lovo cells $\left(2 \times 10^{6}\right)$ were infected with Lenti-Vector or Lenti-S100A16 (S100A16 overexpression vector) and were injected subcutaneously into the flanks of mice to construct the xenograft model ( $n=5$ /group). Tumour volume was measured and recorded every three days. At 21 days after injection, all mice were euthanized via cervical dislocation. The mice were considered to be dead when the heart and breathing stopped. The tumour xenografts were removed to determine their size and weight. The largest tumour diameter was $1.5 \mathrm{~cm}$. All experimental procedures were conducted in accordance with the ethical standards of the Ethical Committee of Nanfang Hospital, Southern Medical University. Study approval was additionally gained from the Institutional Animal Care and Use Committee of Southern Medical University (approval no. L2018160).

Statistical analysis. GraphPad Prism 6 (GraphPad Software, Inc.) and SPSS 22.0 (IBM Corp.) software was used to assess 

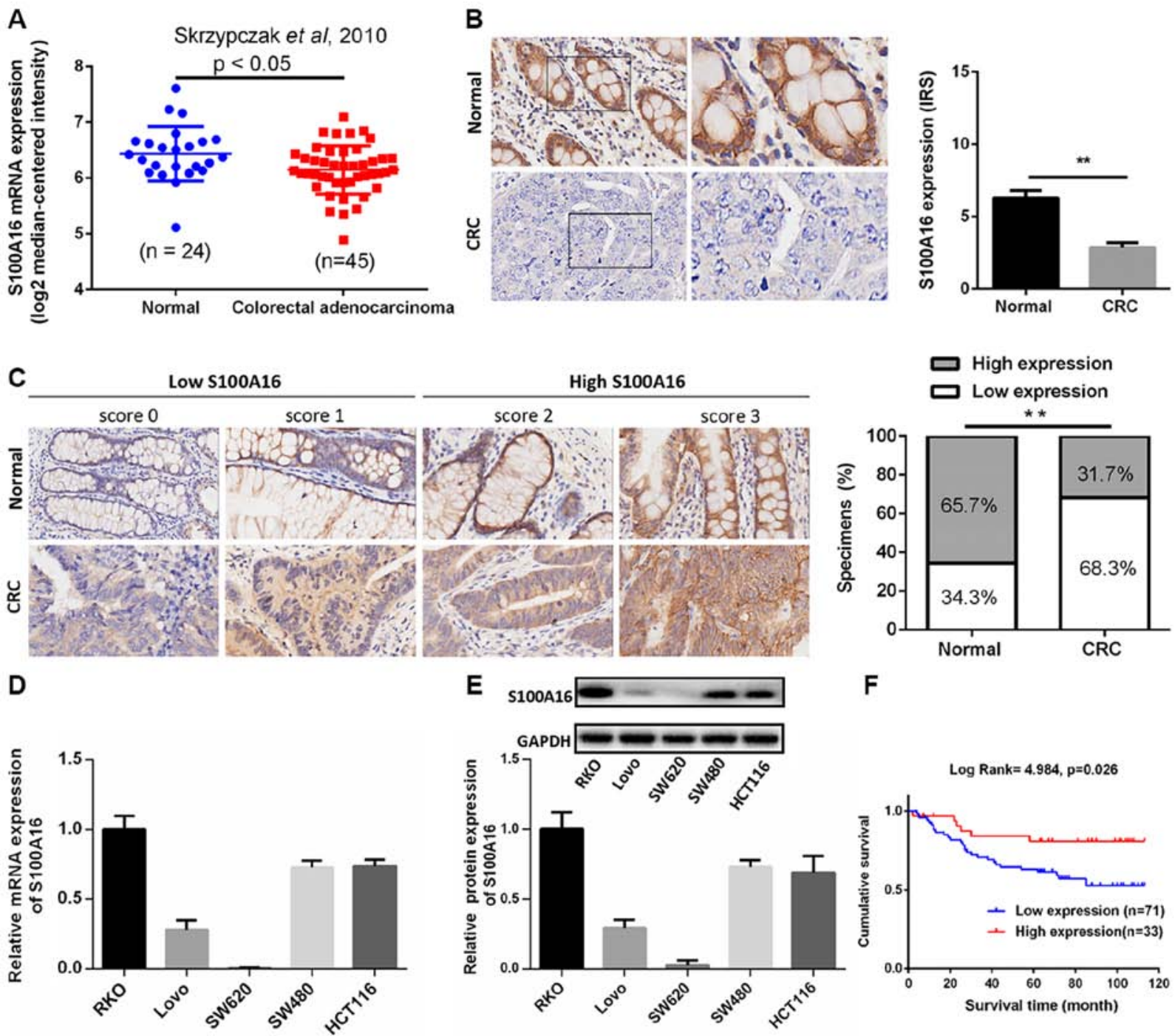

Figure 1. S100A16 is decreased in human colorectal cancer. (A)Analysis of S100A16 expression in normal colorectal tissues and colorectal adenocarcinoma tissues in the Skrzypczak in Oncomine microarray dataset, as assessed using an unpaired-t test. $\mathrm{P}<0.05$. (B) Representative images of S100A16 protein expression in CRC tumour tissues and paired normal adjacent tissues, as assessed via immunohistochemistry. Magnification, $\mathrm{x} 400$. ${ }^{* *} \mathrm{P}<0.01$, paired Student's $\mathrm{t}$-test. (C) Representative images of difference scores indicating S100A16 protein expression in CRC tumour tissues and paired normal adjacent tissues (magnification, $\mathrm{x} 400$ ). The proportion of S100A16-expressing in CRC tissue samples was also analysed. ${ }^{* *} \mathrm{P}<0.01, \chi^{2}$ test. S100A16 (D) mRNA and (E) protein expression in CRC cell lines, as determined via reverse transcription-quantitative PCR and western blotting, respectively. (F) Kaplan-Meier analysis of the survival rates in patients with CRC with S100A16 low or high expression. CRC, colorectal cancer; S100A16, S100 calcium binding protein A16, IRS, immunoreactive score.

the statistical significance of differences between groups. Data were analysed using an unpaired or a paired two-tailed Student's t-test for two groups, or one-way ANOVA followed by Tukey's post hoc test for multiple groups. Two-way ANOVA with Bonferroni's correction was used for statistical analyses between different treatments, different cell cohorts or different time points. $\chi^{2}$ or Fisher's exact tests were used to compare the differences in age, sex, pathological differentiation, T classification, $\mathrm{N}$ classification and American Joint Committee on Cancer (AJCC) stages (Table I). Data are presented as the mean \pm SD from a minimum of three experimental repeats. $\mathrm{P}<0.05$ was considered to indicate a statistically significant difference.

\section{Results}

S100A16 is decreased in human CRC tissues. To identify the potential role of S100A16 in CRC, gene expression profiles were analysed from Oncomine microarray datasets (16). The results revealed that S100A16 mRNA was significantly downregulated in colorectal adenocarcinoma compared with normal tissues (Fig. 1A). The expression of S100A16 was subsequently determined in $70 \mathrm{CRC}$ samples (clinical samples from Nanfang Hospital, $n=10$; paired samples in CRC tissue microarrays purchased from Shanghai Outdo Biotech Co., Ltd., $n=60$ ) and paired normal adjacent tissues. As presented in Fig. 1B, IHC analysis demonstrated that S100A16 exhibited weaker staining in CRC tumour tissues compared with normal tissues. In addition, S100A16 protein was localized to the cell membrane and cytoplasm of CRC cells. To confirm the expression of S100A16 in CRC, CRC microarrays were screened alongside the CRC samples obtained in the present study. The results demonstrated that S100A16 was decreased in CRC tissues compared with normal colon tissues (Fig. 1C).

The expression levels of S100A16 mRNA and protein in five CRC cell lines were examined via RT-qPCR and western blot- 
Table I. Association between clinicopathological features and S100A16 expression in 104 patients with colorectal cancer.

\begin{tabular}{|c|c|c|c|c|c|}
\hline \multirow[b]{2}{*}{ Variables } & \multirow[b]{2}{*}{$\mathrm{n}$} & \multicolumn{2}{|c|}{ S100A16 expression } & \multirow[b]{2}{*}{$\chi^{2}$} & \multirow[b]{2}{*}{ P-value } \\
\hline & & Low $(n=71)$ & High $(n=33)$ & & \\
\hline Age, years & & & & 0.280 & 0.596 \\
\hline$\leq 50$ & 19 & 12 & 7 & & \\
\hline$>50$ & 85 & 59 & 26 & & \\
\hline Sex & & & & 0.000 & 0.987 \\
\hline Female & 44 & 30 & 14 & & \\
\hline Male & 60 & 41 & 19 & & \\
\hline Pathological differentiation & & & & 6.638 & 0.036 \\
\hline I (high) & 11 & 7 & 4 & & \\
\hline II (moderate) & 49 & 28 & 21 & & \\
\hline III (poor) & 44 & 36 & 8 & & \\
\hline $\mathrm{T}$ classification & & & & 0.133 & 0.715 \\
\hline $\mathrm{T} 1+\mathrm{T} 2$ & 6 & 5 & 1 & & \\
\hline $\mathrm{T} 3+\mathrm{T} 4$ & 98 & 66 & 32 & & \\
\hline $\mathrm{N}$ classification & & & & 4.477 & 0.034 \\
\hline No & 60 & 36 & 24 & & \\
\hline $\mathrm{N} 1+\mathrm{N} 2$ & 44 & 35 & 9 & & \\
\hline AJCC stage & & & & 3.311 & 0.069 \\
\hline $\mathrm{I}+\mathrm{II}$ & 59 & 36 & 23 & & \\
\hline III + IV & 45 & 35 & 10 & & \\
\hline
\end{tabular}

S100A16, S100 calcium binding protein A16. AJCC, American Joint Committee on Cancer.

ting, respectively. The results indicated that S100A16 mRNA and protein expression levels were markedly decreased in Lovo and SW620 CRC cell lines (Fig. 1D and E). Among the CRC cell lines assessed in the present study, HCT116 and SW480 cells exhibited higher expression levels of S100A16, and as such, were selected to perform loss-of-function experiments. Lovo cells exhibiting lower expression levels of S100A16 were selected for gain-of-function experiments. As SW620 cells did not express an appropriate level of S100A16, they were not selected for further study.

S100A16 is negatively associated with CRC progression and positively associated with patient survival. To investigate the clinical importance of S100A16 in CRC, the relationship between patient clinicopathological features and S100A16 expression was analysed. Patients with CRC were classified into low and high S100A16 expression groups based on immunostaining scores (16). The results suggested that the expression of S100A16 was closely associated with pathological differentiation $(\mathrm{P}=0.036)$ and $\mathrm{N}$ classification $(\mathrm{P}=0.034)$. However, no association was identified between age, sex, $\mathrm{T}$ classification and AJCC stage (Table I). Furthermore, patients in the low S100A16 expression group demonstrated poor prognosis, with a lower survival time (Fig. 1F). The results indicated that S100A16 may be involved in CRC development and could serve as a potential therapeutic target for CRC.

S100A16 inhibits CRC cell proliferation, migration and invasion. To determine whether S100A16 affects CRC cell proliferation, migration and invasion, siRNAs targeting S100A16 were transfected into HCT116 and SW480 cells. The results demonstrated that S100A16 protein expression was significantly decreased following siRNA transfection. si-S100A16\#1, si-S100A16\#2, and si-S100A16\#3 were all efficiently in silencing S100A16; thus, only two siRNAs were arbitrarily selected for subsequent experiments (Fig. 2A). However, no significant differences were identified in cells transfected with S100A4 and S100A14 siRNA, indicating that S100A16 knockdown was specific to S100A16 (Fig. S1A and B). Subsequently, the S100A16 overexpression vector was transfected into Lovo cells for S100A16 upregulation (Fig. 2B). CCK-8 assays were then performed to determine cell proliferation. The results indicated that proliferation was increased in S100A16-silenced HCT116 and SW480 cells, but decreased in S100A16-overexpressing Lovo cells, compared with the corresponding control groups (Fig. 2C and D). The results of Transwell assays demonstrated that S100A16 knockdown promoted the migration and invasion of HCT116 and SW480 cells, whereas S100A16 overexpression had the opposite effect in Lovo cells (Fig. 3A and B).

S100A16 knockdown activates the JNK/p38 MAPK signalling pathway to promote epithelial-mesenchymal transition (EMT). The signalling pathways associated with the S100A16mediated suppression of CRC cell proliferation, migration and invasion were assessed in the current study. Previous reports have revealed that S100A16 affects the JNK/p38 MAPK signalling pathway $(9,20)$, which serves an important role in 
A
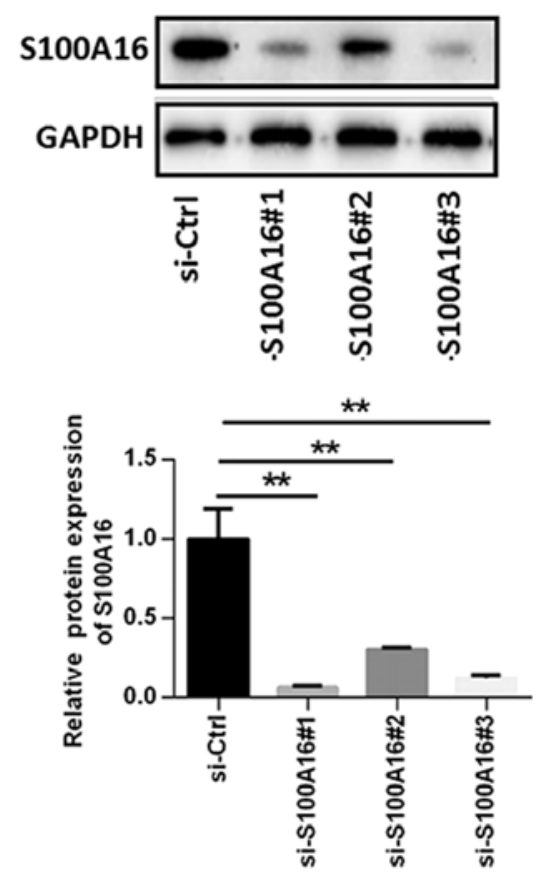

C

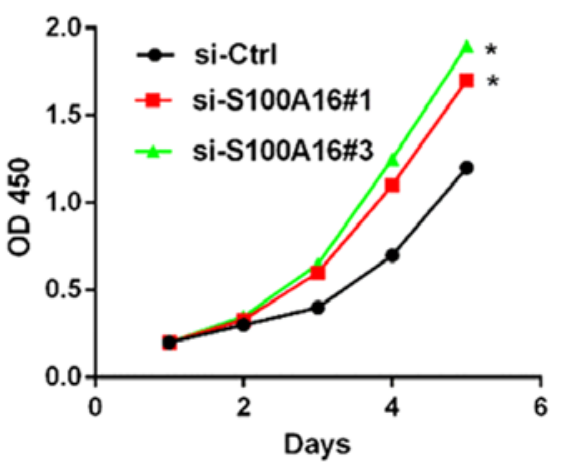

SW480
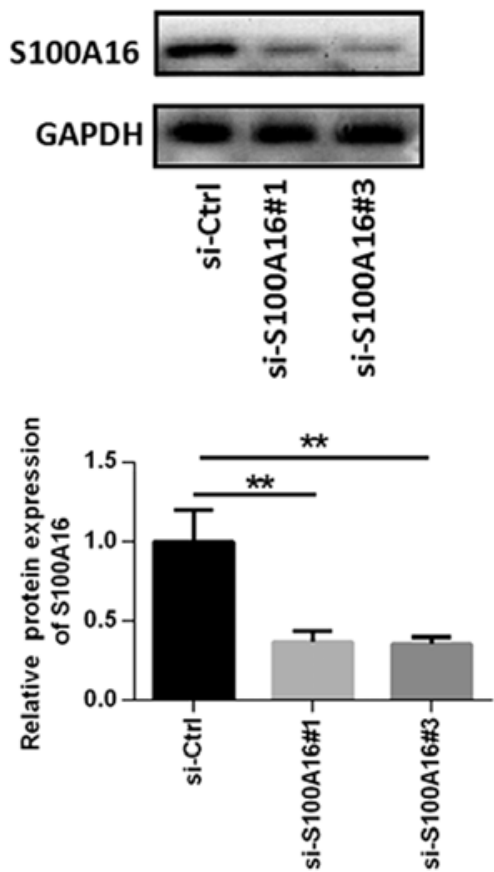

Sw480

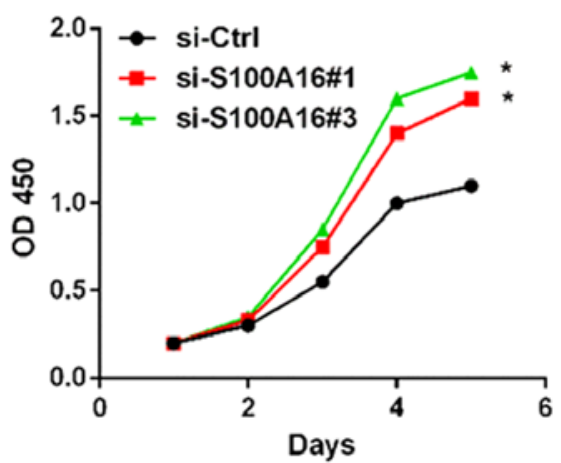

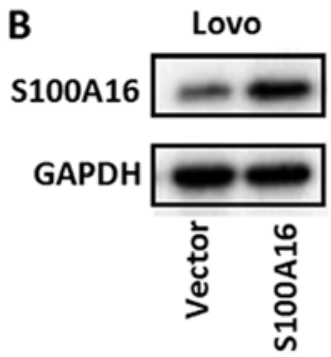

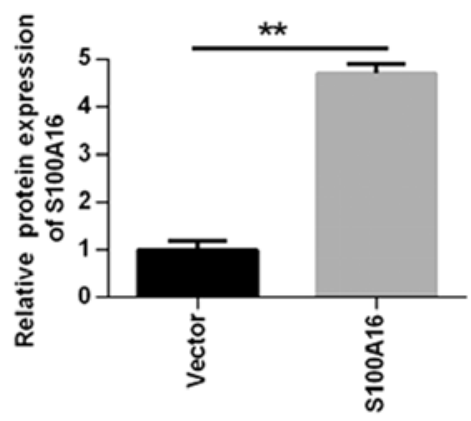

D

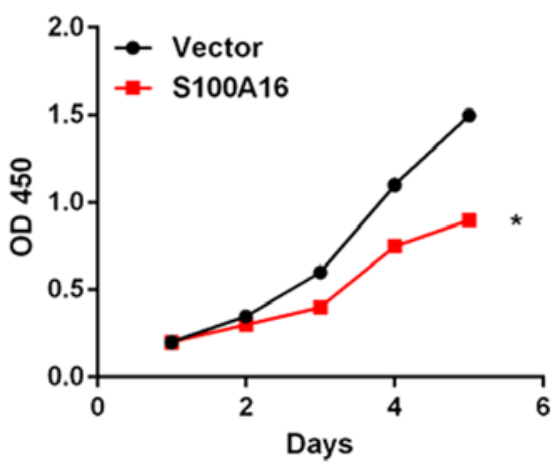

Figure 2. S100A16 inhibits the proliferation of CRC cells. (A) Western blot analysis of S100A16 protein expression in CRC cells transfected with S100A16 siRNAs compared with si-Ctrl. Data were analysed using a one-way ANOVA followed by Tukey's post hoc test. ${ }^{* *} \mathrm{P}<0.01$. (B) Western blot analysis of S100A16 protein expression in CRC cells transfected with overexpression plasmids compared vectors, as assessed using an unpaired Student's t-test. ${ }^{* *} \mathrm{P}<0.01$. (C) S100A16 knockdown promoted the proliferation of HCT116 and SW480 cells. ${ }^{*} \mathrm{P}<0.05$, vs. si-Ctrl. (D) S100A16 overexpression suppressed the proliferation of Lovo cells. ${ }^{*} \mathrm{P}<0.05$. Cell proliferation was examined by performing Cell Counting Kit- 8 assays and analysed using a two-way ANOVA with Bonferronis correction. CRC, colorectal cancer; siRNA/si, small interfering RNA; Ctrl, control; S100A16, S100 calcium binding protein A16; OD, optical density.

CRC (21,22). Thus, western blotting was performed to assess the factors associated with the MAPK signalling pathway in S100A16-inhibited HCT116 cells. The results identified that p38, ERK and JNK phosphorylation levels were increased in HCT116 cells following S100A16 knockdown. However, ERK phosphorylation levels were not as high as those observed for p38 and JNK (Fig. 4). Additionally, S100A16 knockdown increased the expression levels of the mesenchymal markers $\mathrm{N}$-cadherin and vimentin, as well as decreased the expression of the epithelial marker E-cadherin (Fig. 4).

To confirm that the MAPK signalling pathway served a role in S100A16-modulated CRC cellular activity, the JNK inhibitor SP600125 and the p38 inhibitor SB203580 were administered to HCT116 cells. SP600125 and SB203580 significantly repressed the increase in HCT116 cell migration and invasion caused by S100A16 silencing (Fig. 5A). The results of western blotting suggested that SP600125 and SB203580 treatments significantly inhibited the S100A16 knockdown-mediated activation of JNK and p38 in HCT116 cells. SP600125 and SB203580 also suppressed the augmented protein expression levels of $\mathrm{N}$-cadherin and vimentin, and reversed the effect of S100A16 silencing on the protein expression of E-cadherin in HCT116 cells (Fig. 5B). The results suggested that S100A16 suppressed EMT and that S100A16 may exert its effects via the JNK/p38 MAPK signalling pathway.

S100A16 elevation suppresses tumour growth in vivo. To further determine the effects of S100A16 on colorectal cancer in vivo, tumour-bearing mice were constructed. Lenti-Vector and Lenti-S100A16 were transfected into Lovo cells to elevate S100A6 expression (Fig. S1C). The largest tumour diameter observed was $1.5 \mathrm{~cm}$. It was determined that the size of tumours 

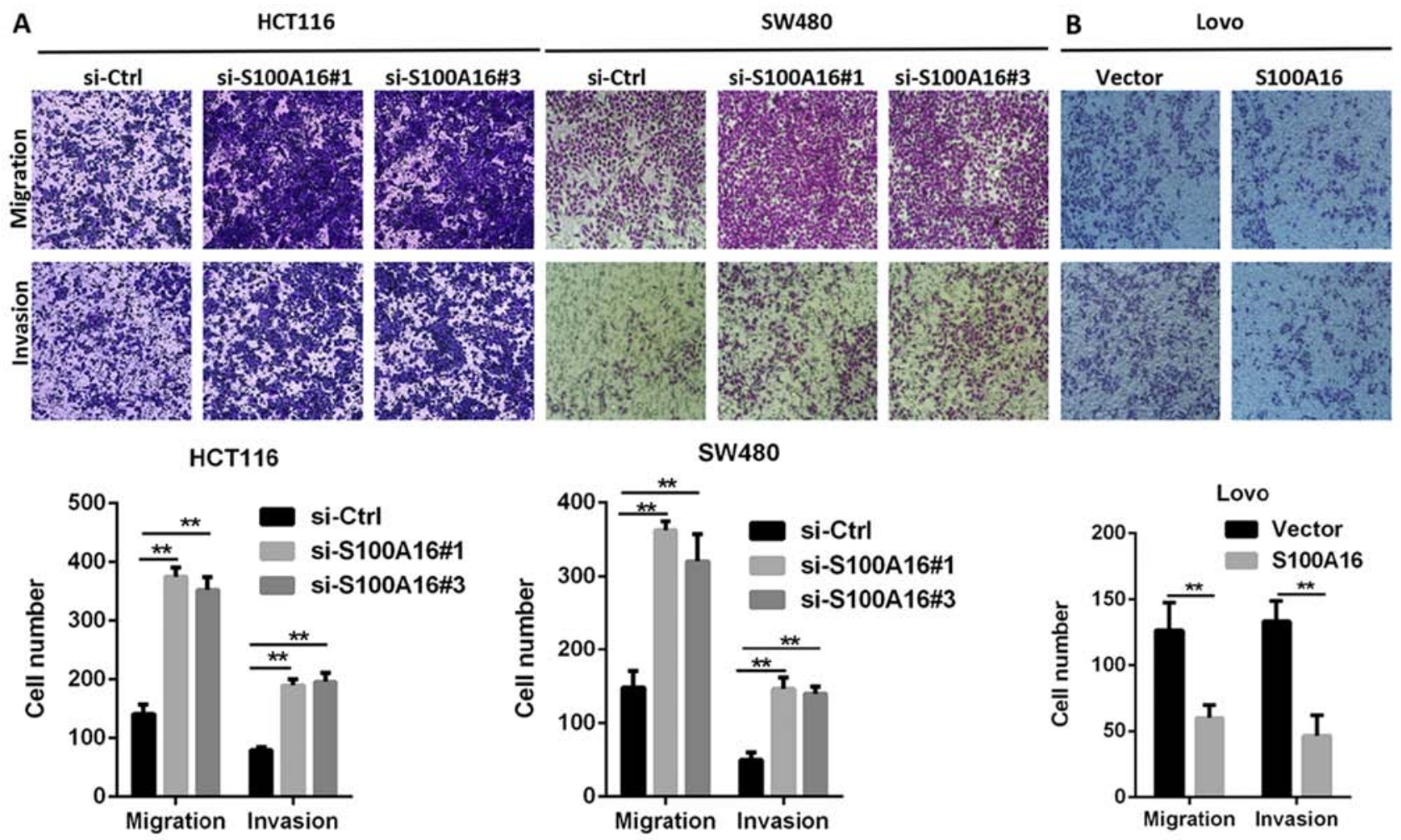

Figure 3. S100A16 inhibits CRC cell migration and invasion. (A) S100A16 knockdown promoted the migration and invasion of the two CRC cell lines, which was analysed using a one-way ANOVA followed by Tukey's post hoc test. ${ }^{* *} \mathrm{P}<0.01$. (B) S100A16 overexpression suppressed the migration and invasion of Lovo cells, which was analysed using an unpaired Student's t-test. ${ }^{* *} \mathrm{P}<0.01$. Cell migration and invasion were determined by performing Transwell assays. CRC, colorectal cancer; S100A16, S100 calcium binding protein A16; siRNA/si, small interfering RNA; Ctrl, control.
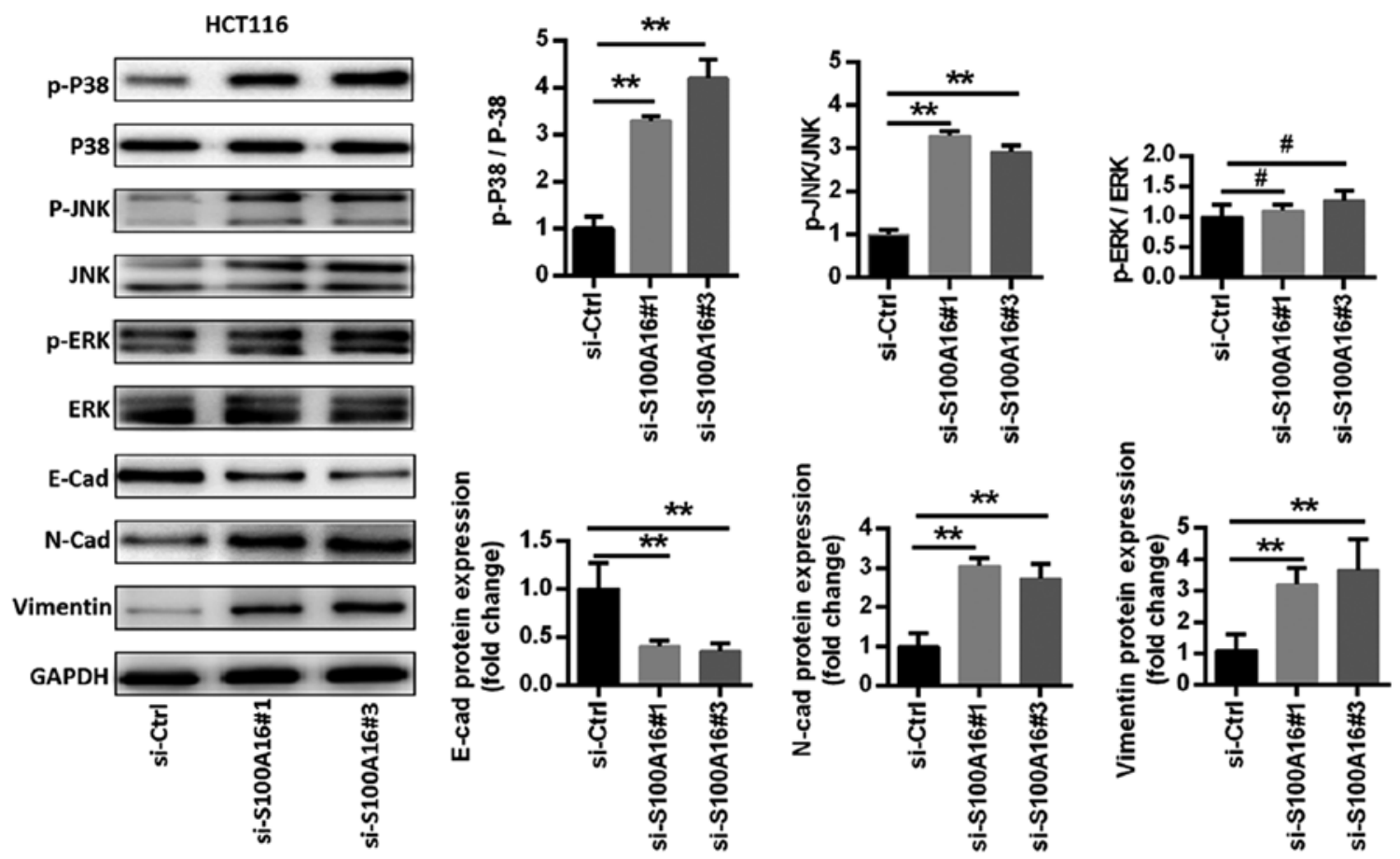

Figure 4. S100A16 knockdown activates the JNK/p38 MAPK signalling pathway and promotes EMT. MAPK pathway-associated and EMT-associated proteins were assessed via western blotting in HCT116 cells. Data were analysed with a one-way ANOVA followed by Tukey's post hoc test. ${ }^{* *} \mathrm{P}<0.01 ;{ }^{~} \mathrm{P}>0.05$ (not significant). EMT, epithelial-mesenchymal transition; S100A16, S100 calcium binding protein A16; p-, phosphorylated; E-cad, E-cadherin; siRNA/si, small interfering RNA; Ctrl, control; N-cad, N-cadherin.

in mice of the S100A16 overexpression group were markedly decreased compared with the control group (Fig. 6A and B).
Additionally, S100A16 overexpression mice exhibited significantly decreased tumour volumes and weights relative to 
A HCT116
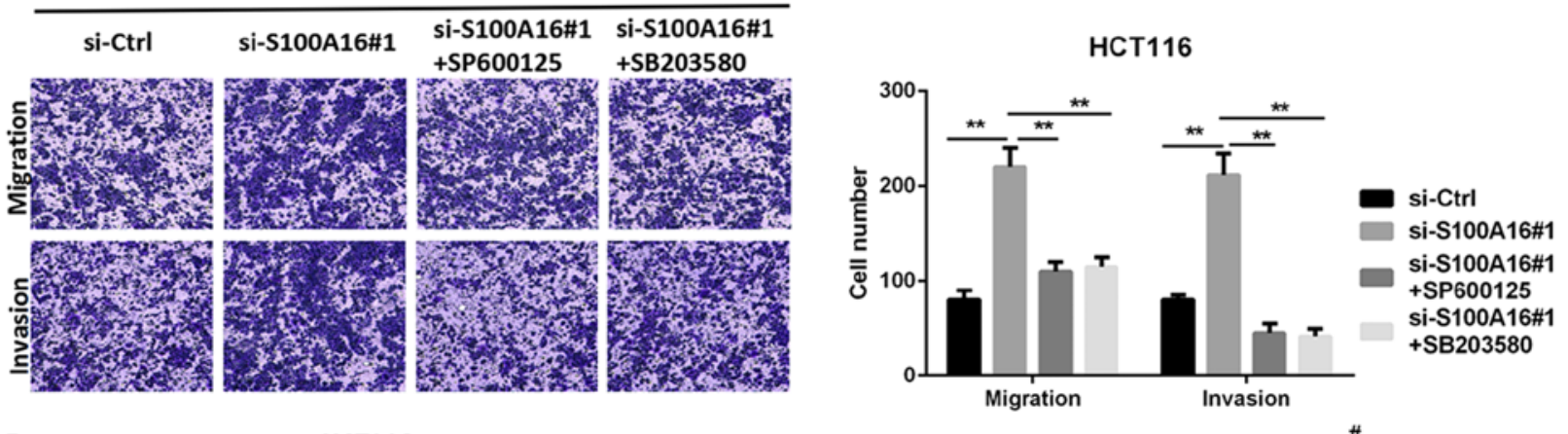

B

HCT116
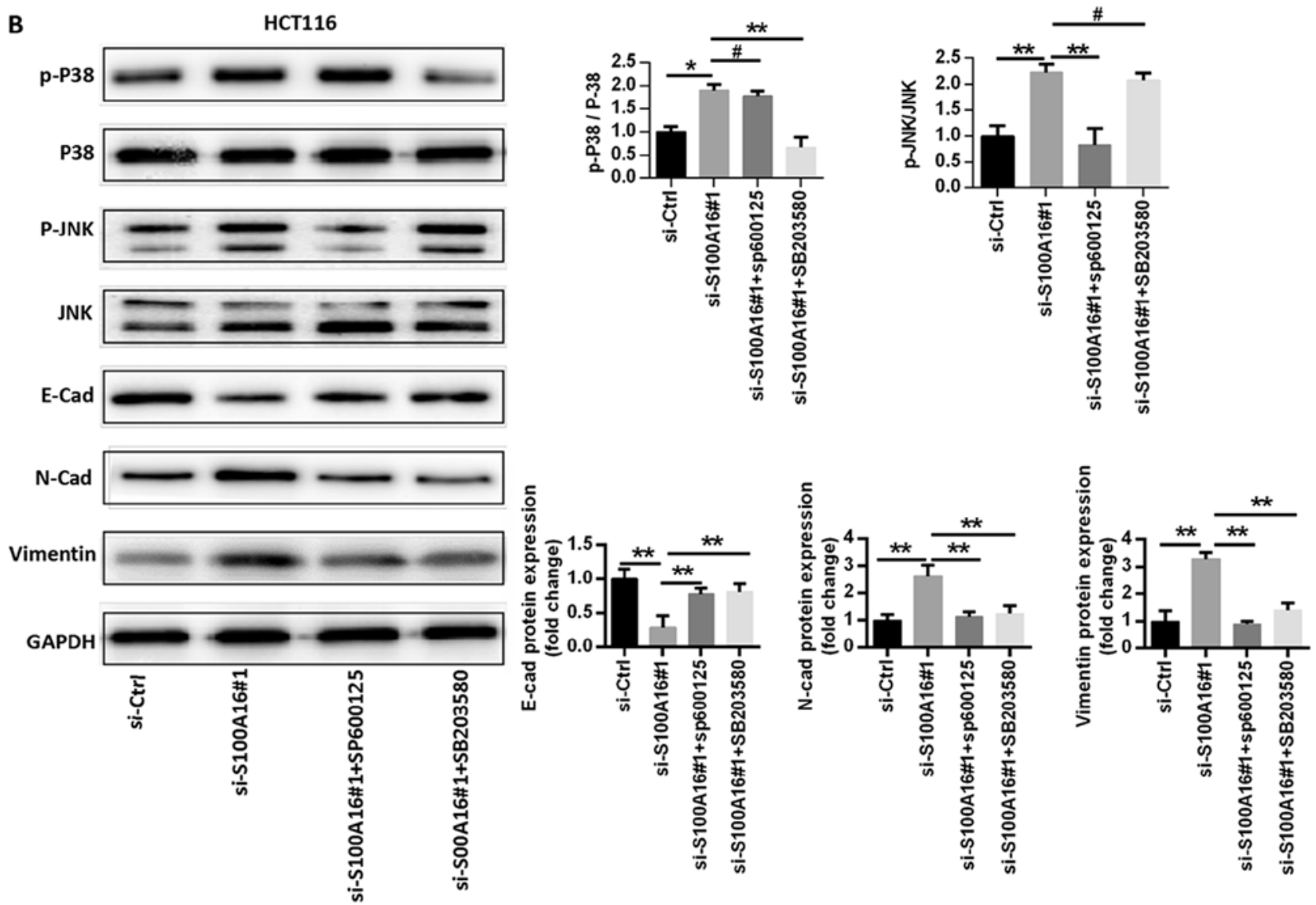

Figure 5. JNK/p38 MAPK signalling pathway inactivation is required for S100A16 knockdown-mediated HCT116 cellular effects. (A) Representative images of Transwell assays after treatment with the p38 inhibitor (SB203580) or the JNK inhibitor (SP600125) following western blotting in S100A16-silenced HCT116 cells. (B) MAPK and epithelial-mesenchymal transition markers were examined via western blotting after treatment with the p38 inhibitor (SB203580) or the JNK inhibitor (SP600125) in S100A16-silenced HCT116 cells. Data were analysed with a one-way ANOVA followed by Tukey's post hoc test. ** P $<0.01$ and ${ }^{*} \mathrm{P}<0.05 ;{ }^{*} \mathrm{P}>0.05$. S100A16, S100 calcium binding protein A16; p-, phosphorylated; E-cad, E-cadherin; siRNA/si, small interfering RNA; Ctrl, control; N-cad, $\mathrm{N}$-cadherin.

the control mice (Fig. 6C and D). The results indicated that S100A16 overexpression inhibited CRC tumours in vivo.

\section{Discussion}

The $\mathrm{S} 100$ family comprises 21 identified $\mathrm{Ca}^{2+}$ binding proteins, several of which are upregulated in various tumours, serving vital roles in tumour progression $(4,23)$. Among these proteins, S100A6 promotes the proliferation and migration of CRC cells (24). Additionally, S100A11 promotes CRC aggressiveness by modulating the TGF $/$ /Smad signalling pathway (25). Previous studies have reported that several S100 members are upregulated in CRC, including S100A2, S100A3, S100A4 and
S100A8, and function as tumour promoters (26-29). In addition, low and high expression levels of S100A14 and S100A4, respectively, are associated with increased CRC metastasis (30). S100A14 interacts with S100A16 and regulates its expression in human cancer cells (31). Furthermore, S100A16 expression is decreased in CRC, which is associated with shorter overall survival times in patients with CRC (15). However, the role of S100A16 in CRC is yet to be fully elucidated. Therefore, the aim of the present study was to determine whether S100A16 serves a functional role in CRC progression.

The present study analysed the expression of S100A16 in the Oncomine microarray dataset, which revealed that S100A16 was downregulated in CRC. The expression of 
A

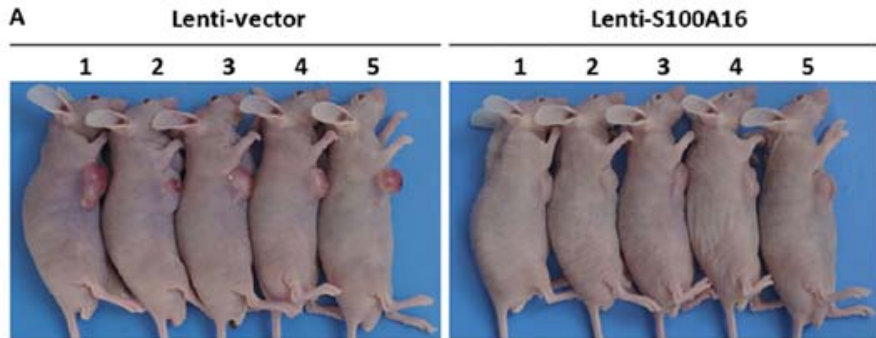

B

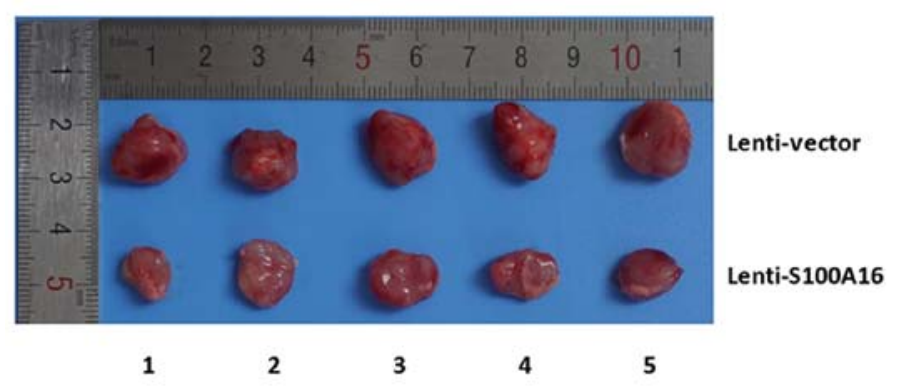

C
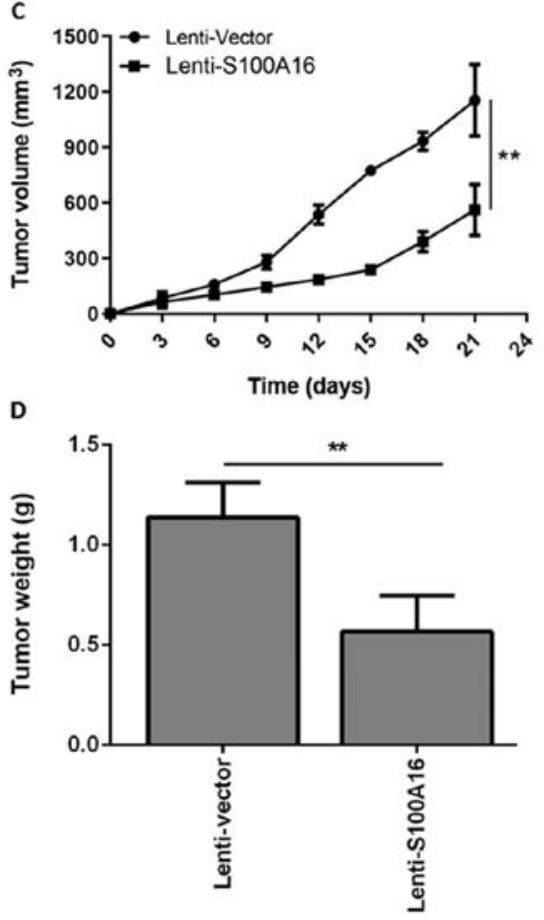

Figure 6. Overexpression of S100A16 inhibits tumour growth in vivo. (A) Images of tumour xenografts in mice of the vector and S100A16 groups. (B) Images of the isolated tumours. (C) Tumour volume and (D) weight were separately compared between the two groups. * P $<0.01$. S100A16, S100 calcium binding protein A16.

S100A16 was further examined in CRC tissue samples and paired adjacent normal tissues via IHC analysis. The results indicated that S100A16 expression was significantly decreased in CRC tumour tissues. The association between patient clinicopathological characteristics and S100A16 expression was subsequently determined. The results demonstrated that S100A16 expression was significantly associated with pathological differentiation and $\mathrm{N}$ classification. Furthermore, decreased S100A16 expression levels were significantly associated with a poor patient prognosis, which is in line with the results of a previous study (15).

Previous studies have revealed that high levels of S100A16 are associated with increased proliferation, migration and invasion $(13,23,32)$. Additionally, S100A16 has been found to affect metastasis (20). However, the effect of S100A16 on the biological activity of CRC cells remains undetermined. The present study therefore investigated the proliferation, migration and invasion of CRC cells in vitro. The results demonstrated that S100A16 knockdown promoted the proliferation, migration and invasion of HCT116 and SW480 cells. By contrast, following S100A16 overexpression, the proliferation, migration and invasion of Lovo cells were decreased. These results indicated that S100A16 may serve as a tumour suppressor in CRC.

Since it has been reported that S100A16 interacts with the MAPK signalling pathway $(9,20)$, which regulates cell proliferation and migration, it was hypothesized that the MAPK signalling pathway may be involved in S100A16-mediated CRC. In the present study, markers of the MAPK signalling pathway were assessed following S100A16 knockdown. It was identified that S100A16 knockdown activated the JNK/p38 MAPK signalling pathway. EMT is a physiological process that increases the migration and invasion of cells, as well as induces tumour metastasis and development $(1,33,34)$. Additionally, EMT is regulated by S100A16 in breast cancer (35). Therefore, the present study assessed EMT markers to investigate the underlying mechanism of CRC progression using MAPK inhibitors following S100A16 knockdown. The results indicated that S100A16 knockdown promoted CRC progression partially via the JNK/P38 MAPK pathway and its subsequent EMT. However, the detailed mechanism via which S100A16 affects the JNK/P38 MAPK pathway was not fully assessed in the present study and may involve additional factors, such as transcription target genes or downstream effectors.

In conclusion, the present study investigated the potential function of S100A16 in CRC, the results of which provided insights into the tumour-suppressive role of S100A16 in CRC cells. S100A16 may serve as a prognostic indicator of CRC, potentially providing a reference for the early diagnosis of CRC, as well as a novel therapeutic target. However, the current study had several limitations. Additional experiments are therefore required to reveal the detailed underlying mechanisms of S100A16 in CRC regulation and should be the focus of future research.

\section{Acknowledgements}

Not applicable.

\section{Funding}

The present study was supported by the National Natural Science Foundation of China (grant nos. 81970451, 81500398 and 81470790), the Natural Science Foundation of Guangdong 
Province (grant nos. 2019A1515010667, 2015A030310480 and 2015A030313295) and the Guangdong Gastrointestinal Disease Research Center (grant no. 2017B02029003).

\section{Availability of data and materials}

The datasets used and/or analysed during the current study are available from the corresponding author on reasonable request.

\section{Authors' contributions}

SO conceived and designed the experiments, performed the experiments, analysed the data, contributed reagents/materials/analytical tools, wrote the manuscript, prepared figures and tables and reviewed the manuscript. YL, JS, JT, YY, FW, WW, JF and FX performed the experiments, analysed the data, contributed reagents/materials/analysis tools and prepared figures and tables. LB conceived and designed the experiments, as well as drafted the manuscript. All authors read and approved the final manuscript.

\section{Ethics approval and consent for publication}

All patients provided written informed consent and the study protocol for the use of clinical samples was approved by The Nanfang Hospital Institutional Review Board (approval no. NFEC-2017-147). Approval for animal studies was additionally gained from The Institutional Animal Care and Use Committee of Southern Medical University (approval no. L2018160).

\section{Patient consent for publication}

Not applicable.

\section{Competing interests}

The authors declare that they have no competing interests.

\section{References}

1. Siegel RL, Miller KD and Jemal A: Cancer statistics, 2019. CA Cancer J Clin 69: 7-34, 2019.

2. Gupta GP and Massagué J: Cancer metastasis: Building a framework. Cell 127: 679-695, 2006.

3. Keane MG and Johnson GJ: Early diagnosis improves survival in colorectal cancer. Practitioner 256: 15-18, 2, 2012.

4. Sturchler E, Cox JA, Durussel I, Weibel M and Heizmann CW: S100A16, a novel calcium-binding protein of the EF-hand superfamily. J Biol Chem 281: 38905-38917, 2006.

5. Donato R: S100: A multigenic family of calcium-modulated proteins of the EF-hand type with intracellular and extracellular functional roles. Int J Biochem Cell Biol 33: 637-668, 2001.

6. Sedaghat F and Notopoulos A: S100 protein family and its application in clinical practice. Hippokratia 12: 198-204, 2008.

7. Heizmann CW: The multifunctional S100 protein family. Methods Mol Biol 172: 69-80, 2002

8. Domínguez B, Pardo BG, Noia M, Millán A, Gómez-Tato A, Martínez P, Leiro J and Lamas J: Microarray analysis of the inflammatory and immune responses in head kidney turbot leucocytes treated with resveratrol. Int Immunopharmacol 15: 588-596, 2013

9. Li D, Zhang R, Zhu W, Xue Y, Zhang Y, Huang Q, Liu M and Liu Y: S100A16 inhibits osteogenesis but stimulates adipogenesis. Mol Biol Rep 40: 3465-3473, 2013.
10. Babini E, Bertini I, Borsi V, Calderone V, Hu X, Luchinat C and Parigi G: Structural characterization of human S100A16, a lowaffinity calcium binder. J Biol Inorg Chem 16: 243-256, 2011.

11. Wang C, Zhu X, Li A, Yang S, Qiao R and Zhang J: S100A16 regulated by Snail promotes the chemoresistance of nonmuscle invasive bladder cancer through the AKT/Bcl-2 pathway. Cancer Manag Res 11: 2449-2456, 2019.

12. Chen, Luo L and Liang C: Aberrant S100A16 expression might be an independent prognostic indicator of unfavorable survival in non-small cell lung adenocarcinoma. PLoS One 13: e197402, 2018.

13. Zhou W, Pan H, Xia T, Xue J, Cheng L, Fan P, Zhang Y, Zhu W, Xue Y, Liu X, et al: Up-regulation of S100A16 expression promotes epithelial-mesenchymal transition via Notch1 pathway in breast cancer. J Biomed Sci 21: 97, 2014.

14. Sapkota D, Bruland O, Parajuli H, Osman TA, Teh MT, Johannessen AC and Costea DE: S100A16 promotes differentiation and contributes to a less aggressive tumor phenotype in oral squamous cell carcinoma. BMC Cancer 15: 631, 2015.

15. Sun X, Wang T, Zhang C, Ning K, Guan ZR, Chen SX, Hong TT and Hua D: S100A16 is a prognostic marker for colorectal cancer. J Surg Oncol 117: 275-283, 2018.

16. Skrzypczak M, Goryca K, Rubel T, Paziewska A, Mikula M, Jarosz D, Pachlewski J, Oledzki J and Ostrowski J: Modeling oncogenic signaling in colon tumors by multidirectional analyses of microarray data directed for maximization of analytical reliability. PLoS One 5: 5, 2010.

17. Shi J, Sun S, Liao Y, Tang J, Xu X, Qin B, Qin C, Peng L, Luo M, Bai L, et al: Advanced oxidation protein products induce G1 phase arrest in intestinal epithelial cells via a RAGE/CD36-JNKp27kip1 mediated pathway. Redox Biol 25: 101196, 2019.

18. Tang J, Liao Y, He S, Shi J, Peng L, Xu X, Xie F, Diao N, Huang J, Xie $\mathrm{Q}$, et al: Autocrine parathyroid hormone-like hormone promotes intrahepatic cholangiocarcinoma cell proliferation via increased ERK/JNK-ATF2-cyclinD1 signaling. J Transl Med 15: $238,2017$.

19. Livak KJ and Schmittgen TD: Analysis of relative gene expression data using real-time quantitative PCR and the 2(-Delta Delta C(T)) Method. Methods 25: 402-408, 2001.

20. Zhu W, Xue Y, Liang C, Zhang R, Zhang Z, Li H, Su D, Liang X, Zhang Y, Huang Q, et al: S100A16 promotes cell proliferation and metastasis via AKT and ERK cell signaling pathways in human prostate cancer. Tumour Biol 37: 12241-12250, 2016.

21. Yang MH, Zhao L, Wang L, Ou-Yang W, Hu SS, Li WL, Ai ML, Wang YQ, Han Y, Li TT, et al: Nuclear lncRNA HOXD-AS1 suppresses colorectal carcinoma growth and metastasis via inhibiting HOXD3-induced integrin $\beta 3$ transcriptional activating and MAPK/AKT signalling. Mol Cancer 18: 31, 2019.

22. Schroyer AL, Stimes NW, Abi Saab WF and Chadee DN: MLK 3 phosphorylation by ERK $1 / 2$ is required for oxidative stress-induced invasion of colorectal cancer cells. Oncogene 37: 1031-1040, 2018.

23. Marenholz I and Heizmann CW: S100A16, a ubiquitously expressed EF-hand protein which is up-regulated in tumors. Biochem Biophys Res Commun 313: 237-244, 2004.

24. Duan L, Wu R, Zou Z, Wang H, Ye L, Li H, Yuan S, Li X, Zha H, Sun H, et al: S100A6 stimulates proliferation and migration of colorectal carcinoma cells through activation of the MAPK pathways. Int J Oncol 44: 781-790, 2014.

25. Niu Y, Shao Z, Wang H, Yang J, Zhang F, Luo Y, Xu L, Ding Y and Zhao L: LASP1-S100A11 axis promotes colorectal cancer aggressiveness by modulating TGF $\beta /$ Smad signaling. Sci Rep 6: 26112, 2016.

26. Masuda T, Ishikawa T, Mogushi K, Okazaki S, Ishiguro M, Iida S, Mizushima H, Tanaka H, Uetake H and Sugihara K: Overexpression of the S100A2 protein as a prognostic marker for patients with stage II and III colorectal cancer. Int J Oncol 48: 975-982, 2016.

27. Liu B, Sun WY, Zhi CY, Lu TC, Gao HM, Zhou JH, Yan WQ and Gao HC: Role of S100A3 in human colorectal cancer and the anticancer effect of cantharidinate. Exp Ther Med 6: 1499-1503, 2013.

28. Dahlmann M, Okhrimenko A, Marcinkowski P, Osterland M, Herrmann P, Smith J, Heizmann CW, Schlag PM and Stein U: RAGE mediates S100A4-induced cell motility via MAPK/ERK and hypoxia signaling and is a prognostic biomarker for human colorectal cancer metastasis. Oncotarget 5: 3220-3233, 2014.

29. Zha H, Sun H, Li X, Duan L, Li A, Gu Y, Zeng Z, Zhao J, Xie J, Yuan S, et al: S100A8 facilitates the migration of colorectal cancer cells through regulating macrophages in the inflammatory microenvironment. Oncol Rep 36: 279-290, 2016. 
30. Wang HY, Zhang JY, Cui JT, Tan XH, Li WM, Gu J and Lu YY: Expression status of S100A14 and S100A4 correlates with metastatic potential and clinical outcome in colorectal cancer after surgery. Oncol Rep 23: 45-52, 2010.

31. Sapkota D, Costea DE, Ibrahim SO, Johannessen AC and Bruland O: S100A14 interacts with S100A16 and regulates its expression in human cancer cells. PLoS One 8: e76058, 2013.

32. Kobayashi M, Nagashio R, Saito K, Aguilar-Bonavides C, Ryuge S, Katono K, Igawa S, Tsuchiya B, Jiang SX, Ichinoe M, et al: Prognostic significance of S100A16 subcellular localization in lung adenocarcinoma. Hum Pathol 74: 148-155, 2018.

33. Brabletz T, Kalluri R, Nieto MA and Weinberg RA: EMT in cancer. Nat Rev Cancer 18: 128-134, 2018.
34. Zeindl-Eberhart E, Brandl L, Liebmann S, Ormanns S, Scheel SK, Brabletz T, Kirchner T and Jung A: Epithelial-mesenchymal transition induces endoplasmic-reticulum-stress response in human colorectal tumor cells. PLoS One 9: e87386, 2014.

35. Tanaka M, Ichikawa-Tomikawa N, Shishito N, Nishiura K, Miura T, Hozumi A, Chiba H, Yoshida S, Ohtake T and Sugino T: Co-expression of S100A14 and S100A16 correlates with a poor prognosis in human breast cancer and promotes cancer cell invasion. BMC Cancer 15: 53, 2015.

(c) () $(9$ This work is licensed under a Creative Commons

cc) Attribution-NonCommercial-NoDerivatives 4.0 International (CC BY-NC-ND 4.0) License. 